\title{
Manuseio do canal arterial patente no prematuro com síndrome de angústia respiratória: ligadura ou indometacina?
}

Milton A. MEIER*, Waldir JAZBIK**, Joaquim H. COUTINHO**, João Carlos JAZBIK**, José Aldrovando de OLIVEIRA ${ }^{\star \star \star}$, José Caetano SILVA ${ }^{\star \star \star}$, Rosa Célia BARBOSA ${ }^{\star \star \star}$, Helder PAUPÉRIO ${ }^{\star \star \star}$, Astolfo SERRA Jr. ${ }^{* \star *}$

MEIER, M. A.; JAZBIK, W.; COUTINHO, J. H.; JAZBIK, J. C.; OLIVEIRA, J. A,; SILVA, J. C.; BARBOSA, R. C.; PAUPÉRIO, H.; SERRA Jr., A. - Manuseio do canal arterial patente no prematuro com sindrome de angústia respiratória: ligadura ou indometacina? Rev. Bras. Cir. Cardiovasc., 4(1): 9-20, 1989.

RESUMO: A presença de canal arterial patente (PCA) com grande shunt sistêmico -pulmonar no prematuro com síndrome de angústia respiratória (SAR) está quase sempre associada a insuficiência cardiaca, displasia broncopulmonar, enterocolite necrotizante, hemorragia intracraniana e morte. A ligadura do canal melhora a complacência pulmonar, reduz, significativamente, o tempo de assistência ventilatória e melhora o estado geral do paciente. Depois da introduçăo, nesta última década, da indometacina para a interrupção do PCA, no prematuro, vários estudos vêm procurando estabelecer as vantagens de uma forma de tratamento sobre a outra. O propósito do nosso estudo é comparar os resultados obtidos em 48 pacientes (pts.) (Grupo 1) submetidos a ligadura cirúrgica, nos últimos 8 anos, com 28 pts. (Grupo 2) nos quais a indometacina foi, preferentemente, utilizada. A idade gestacional média do Grupo 1 foi de 29,13 $\pm 2,33$ semanas (24-34) e de $28,39 \pm 2,30$ semanas (25-32) no Grupo 2. O peso médio foi de $954,17 \pm 220,68 \mathrm{~g}(540-1750 \mathrm{~g})$ no Grupo 1 e de $923,21 \pm 191,74 \mathrm{~g}(550-1400 \mathrm{~g})$ no Grupo 2. Trinta e três $(60,75 \%)$ dos pts. do Grupo 1 eram menores do que $1000 \mathrm{~g}$ (prematuros extremos), enquanto que 21 do Grupo 2 (75,0\%) estavam nessa condição. Nove pacientes $(32,1 \%)$ do Grupo 2 foram transferidos para o Grupo 1, devido ao insucesso da terapêutica com a indometacina, ou pela intolerância às doses indicadas. A ligadura foi realizada na própria unidade de terapia intensiva em 31 pts. $(64,5 \%)$. A técnica empregada tem sido a de uma toracotomia póstero-lateral pequena, com preservação dos músculos do tórax e abertura pelo $3^{\circ}$ ou 4 : espaço intercostal. Por via extrapleural, o canal é alcançado e ligado com dois clips metálicos. Inicialmente, o tórax era rotineiramente drenado, apenas quando havia abertura da pleura. Mais recentemente, mesmo com a pleura aberta, somente são drenados os casos que apresentam pneumotórax prévio, ou então sangramento excessivo. A mortalidade no Grupo 1 foi de $18,75 \%$ (9 pts.) e, no Grupo 2, de 25,0\% (7 pts.). Quatro pacientes do Grupo 2 transferidos para o Grupo 1 faleceram. As causas de óbito no Grupo 1 foram sépsis e hemorragia intracraniana; no Grupo 2, sépsis, enterocolite necrotizante com perfuração localizada, hemorragia intracraniana e penumotórax. Nos dois grupos, a mortalidade foi significantemente maior nos prematuros extremos. A despeito dos problemas de comparação entre os 2 grupos e das conclusōes limitadas que podem ser retiradas de um estudo retrospectivo e não randomizado, acreditamos que os resultados da ligadura cirúrgica são nitidamente superiores aos obtidos com o uso da indometacina

DESCRITORES: persistência de canal arterial, cirurgia.

Trabalho realizado no Hospital Universitário Pedro Ernesto da Universidade Estadual do Rio de Janeiro e no Instituto Estadual de Cardiologia Aloysio de Castro. Rio de Janeiro. RJ. Brasil.

Apresentado ao 16: Congresso Nacional de Cirurgia Cardiaca. São Paulo, 7 e 8 de abril de 1989.

* Do Hospital Universitário Pedro Ernesto e do Instituto Estadual de Cardiologia Aloysio de Castro.

** Do Hospital Universitário Pedro Ernesto

*** Do Instituto Estadual de Cardiologia Aloysio de Castro.

Endereço para separatas: Milton A. Meier. Rua Almirante Alexandrino, 1.908, apto. 102.20241 Rio de Janeiro, RJ, Brasil 
MEIER, M. A.; JAZBIK, W.; COUTINHO, J. H.; JAZBIK, J. C.; OLIVEIRA, J. A.; SILVA, J. C.; BARBOSA, R. C.; PAUPÉRIO, H.; SERRA Jr., A. - Manuseio do canal arterial patente no prematuro com síndrome de angústia respiratória: ligadura ou indometacina? Rev. Bras. Cir. Cardiovasc., 4(1): 9-20, 1989.

\section{INTRODUÇÃO}

O canal arterial patente com grande shunt sistêmicopulmonar, no prematuro e prematuro extremo com síndrome de angústia respiratória (SAR), causa, freqüentemente, insuficiência cardiaca congestiva e está associado com displasia broncopulmonar, enterocolite necrotizante, hemorragia intracraniana e taxa elevada de óbitos $^{3}, 7,13,15,30,32,53,56,59$. A Interrupção desse shunt melhora a complacência pulmonar, diminui o tempo de assistência ventilatória e melhora a utilização nutricional $^{12,46}$. Essa interrupção pode ser realizada cirurgicamente, pela ligadura, ou farmacologicamente, pela administração de um inibidor das prostaglandinas, a indometacina. O uso da indometacina para a constricçāo farmacológica do canal arterial teve início em $1976^{19}, 20,27$. Logo, uma série de estudos foram publicados ${ }^{36}$. 40, 49, 61, mostrando que, se não houvesse contra-indicaçōes, essa substância seria eficaz para fechar o canal arterial em prematuros. Contudo, nem sempre a indometacina é eficiente ${ }^{1,29}$ e, além disto, uma série de complicações começaram a ser relatadas na literatura ${ }^{18,25,44,47}, 0$ que levou a um maior cuidado nas indicaçōes dessa droga, até que resultados a longo prazo pudessem ser avaliados. Um desses trabalhos ${ }^{45}$ mostrou, especificamente, que a indometacina causa, com freqüência significativa, enterocolite necrotizante, perfuração intestinal e diminuí, quase sempre, a função renal. Entre as muitas contra-indicaçōes do emprego da indometacina, estāo a hiperbilirrubinemia, sepsis, deficiências de coagulação sangüínea e insuficiência renal ${ }^{13}$. 0 propósito do nosso estudo é comparar os resultados obtidos com a ligadura cirúrgica do canal arterial em prematuros e prematuros extremos com síndrome de angústia respiratória (SAR), com o mesmo tipo de pacientes que receberam indometacina para obter o fechamento farmacológico do canal.

\section{CASUISTICA E MÉTODOS}

Os pacientes que constituem o objetivo deste estudo são 48 prematuros submetidos a ligadura cirúrgica do canal arterial, Grupo 1 (Tabela 1), e 28 prematuros nos quais a interrupçăo do canal foi tentada com indometacina, Grupo 2 (Tabela 2). Os 2 grupos são de prematuros internados em várias unidades de neonatologia do Rio de Janeiro*, num período de 8 anos e analisados retrospectivamente. Todos os pacientes foram transferidos para essas unidades após a constatação da SAR, nos berçários de origem. Outros 5 pacientes, que também foram submetidos a ligadura cirúrgica do canal, e 8, que foram tratados com indometacina, foram excluidos destas duas séries, por causa de prontuários incompletos, o que impossibilitava o estudo.

\footnotetext{
- Hospital Universitário Pedro Emesto (UERJ), Centro de Prematuros do Rio de Janeiro (CEPERJ), Centro de Neonatologia Lagoa (URGIL), Unidade de Neonatologia da URPE, Unidade de Neonatologia da Casa de Saúde São Vicente, Pronto-Socorro Infantil Lagoa (PSIL) e Hospital Fernandes Filgueiras (MS).
}

TABELA 1

PERSISTENNCIA DE CANAL ARTERIAL - GRUPO 1

\begin{tabular}{|c|c|c|c|c|}
\hline N: & $\begin{array}{c}\text { Peso } \\
\text { (g) }\end{array}$ & $\begin{array}{c}\text { Idade } \\
\text { Gestacional } \\
\text { (Sem.) }\end{array}$ & $\begin{array}{c}\text { Idade Extra } \\
\text { Uterina } \\
\text { (Dias) }\end{array}$ & $\begin{array}{l}\text { Resultado } \\
\text { Altal Obito }\end{array}$ \\
\hline 1 & 1750 & 33 & 27 & $\bullet$ \\
\hline 2 & 1200 & 33 & 22 & $\star$ \\
\hline 3 & 950 & 29 & 14 & $\star$ \\
\hline 4 & 1080 & 32 & 24 & $*$ \\
\hline 5 & 1150 & 31 & 18 & $\star$ \\
\hline 6 & 960 & 28 & 12 & $\star$ \\
\hline 7 & 1050 & 30 & 15 & $\star$ \\
\hline 8 & 950 & 27 & 18 & + \\
\hline 9 & 910 & 28 & 20 & $\star$ \\
\hline 10 & 1080 & 31 & 14 & $\star$ \\
\hline 11 & 820 & 29 & 8 & + \\
\hline 12 & 840 & 28 & 11 & $\star$ \\
\hline 13 & 910 & 29 & 18 & $\star$ \\
\hline 14 & 760 & 28 & 6 & + \\
\hline 15 & 890 & 28 & 12 & $*$ \\
\hline 16 & 1120 & 32 & 27 & $\star$ \\
\hline 17 & 1250 & 32 & 45 & $\cdot$ \\
\hline 18 & 550 & 25 & 5 & + \\
\hline 19 & 970 & 29 & 17 & $*$ \\
\hline 20 & 770 & 26 & 11 & $\star$ \\
\hline 21 & 860 & 28 & 16 & $\star$ \\
\hline 22 & 950 & 30 & 9 & $*$ \\
\hline 23 & 1350 & 34 & 22 & $\star$ \\
\hline 24 & 740 & 26 & 10 & + \\
\hline 25 & 980 & 30 & 19 & $\star$ \\
\hline 26 & 990 & 30 & 21 & $\star$ \\
\hline 27 & 540 & 24 & 21 & $*$ \\
\hline 28 & 890 & 28 & 17 & + \\
\hline 29 & 940 & 30 & 19 & $*$ \\
\hline 30 & 1000 & 31 & 8 & $\star$ \\
\hline 31 & 950 & 30 & 21 & $*$ \\
\hline 32 & 1100 & 32 & 16 & $\star$ \\
\hline 33 & 900 & 30 & 8 & * \\
\hline 34 & 840 & 29 & 10 & * \\
\hline 35 & 1550 & 33 & 10 & * \\
\hline 36 & 790 & 27 & 14 & $*$ \\
\hline 37 & 800 & 28 & 8 & + \\
\hline 38 & 1080 & 31 & 9 & $*$ \\
\hline 39 & 1040 & 32 & 8 & + \\
\hline 40 & 950 & 28 & 10 & $*$ \\
\hline 41 & 960 & 27 & 22 & $\star$ \\
\hline 42 & 840 & 26 & 14 & $*$ \\
\hline 43 & 1000 & 29 & 12 & $\star$ \\
\hline 44 & 720 & 28 & 18 & * \\
\hline 45 & 850 & 29 & 19 & $*$ \\
\hline 46 & 630 & 25 & 13 & $\star$ \\
\hline 47 & 660 & 26 & 20 & + \\
\hline 48 & 940 & 29 & 25 & $\star$ \\
\hline
\end{tabular}

A idade gestacional média do Grupo 1 foi de 29,13 $\pm 2,33$ semanas ( $24-33$ semanas) e de $28,39 \pm 2,30$ semanas (25-32 semanas) no Grupo 2. O peso médio foi de $954,17 \pm 220,86 \mathrm{~g}(540-1750 \mathrm{~g})$ no grupo $1 \mathrm{e}$ de $923,21 \pm 191,74 \mathrm{~g}(540-1400 \mathrm{~g})$ no Grupo 2 . Trinta e três $(68,75 \%)$ pacientes (pts.) do Grupo 1 eram meno- 
MEIER, M. A.; JAZBIK, W.; COUTINHO, J. H.; JAZBIK, J. C.; OLIVEIRA, J. A.; SILVA, J. C.; BARBOSA, R. C.; PAUPÉRIO, H.; SERRA Jr., A. - Manuseio do canal arterial patente no prematuro com síndrome de angústia respiratória: ligadura ou indometacina? Rev. Bras. Cir. Cardiovasc., 4(1): 9-20, 1989.

TABELA 2

PERSISTENCIA DE CANAL

ARTERIAL - GRUPO 2

\begin{tabular}{|c|c|c|c|c|}
\hline$N^{0}$ & $\begin{array}{l}\text { Peso } \\
\text { (g) }\end{array}$ & $\begin{array}{l}\text { Idade } \\
\text { Gestacional } \\
\text { (Sem.) }\end{array}$ & $\begin{array}{c}\text { Idade Extra } \\
\text { Uterina } \\
\text { (Dias) }\end{array}$ & $\begin{array}{l}\text { Resultado } \\
\text { Alta Obito }\end{array}$ \\
\hline 1 & 890 & 28 & 5 & * \\
\hline 2 & 950 & 29 & 16 & * \\
\hline 3 & 1150 & 32 & 19 & * \\
\hline 4 & $960^{\circ}$ & 28 & 12 & * \\
\hline 5 & 790 & 26 & 27 & + \\
\hline 6 & 930 & 28 & 14 & $*$ \\
\hline 7 & $820^{\circ}$ & 29 & 8 & + \\
\hline 8 & 1120 & 31 & 28 & * \\
\hline 9 & 830 & 25 & 13 & + \\
\hline 10 & $910^{\circ}$ & 29 & 18 & * \\
\hline 11 & 790 & 25 & 11 & + \\
\hline 12 & 650 & 25 & 9 & + \\
\hline 13 & $550^{\circ}$ & 25 & 5 & + \\
\hline 14 & 1400 & 32 & 27 & $\star$ \\
\hline 15 & 990 & 30 & 22 & $*$ \\
\hline 16 & 950 & 28 & 29 & * \\
\hline 17 & $740^{\circ}$ & 26 & 10 & + \\
\hline 18 & 1250 & 31 & 14 & \\
\hline 19 & 960 & 29 & 12 & + \\
\hline 20 & 880 & 28 & 15 & $\star$ \\
\hline 21 & $950^{\circ}$ & 30 & 7 & $\star$ \\
\hline 22 & 1090 & 31 & 36 & * \\
\hline 23 & 960 & 29 & 24 & + \\
\hline 24 & $1040^{\circ}$ & 32 & 8 & * \\
\hline 25 & 1150 & 31 & 7 & * \\
\hline 26 & 630 & 25 & 13 & * \\
\hline 27 & $660^{\circ}$ & 26 & 20 & + \\
\hline 28 & 860 & 28 & 21 & * \\
\hline
\end{tabular}

- Transferidos para o Grupo 1 (9 pts., 32,1\%)

- Sobrevivente

+Óbito

TABELA 3

CONTRA-INDICACÓES DA

INDOMETACINA EM PREMATUROS

COM PCA

Uréia $\geqslant 30 \mathrm{mg} / \mathrm{dl}$

Creatinina $\geqslant 1,8 \mathrm{mg} / \mathrm{dl}$

Volume urinário $<0,6 \mathrm{ml}$, kg. hr nas $8 \mathrm{hs}$ precedentes

Plaquetas $<60.000 / \mathrm{cu} \mathrm{mm}$

Sangue oculto nas fezes $>3+$ (ou moderado para grande)

Evidência de diátese hemorrágica

Evidências clínicas ou radiológicas de enterocolite necrotizante

Evidências clínicas ou nāo-invasivas de hemorragia intracraniana nos últimos 7 dias.

res de $1000 \mathrm{~g}$ (prematuros extremos), enquanto que 21 do Grupo $2(75,0 \%)$ estavam nessa condição. Nove pacientes do Grupo $2(32,1 \%)$ foram transferidos para o Grupo 1, devido ao insucesso da terapêutica com a indometacina, ou por não tolerarem as doses indicadas, ou, ainda, por reabertura do canal.
Todos os pacientes apresentavam a SAR, estavam com tubo endotraqueal e com assistência ventilatória e mostravam sinais de insuficiência cardiaca congestiva. O canal arterial patente foi constatado por uma ou mais das seguintes técnicas: exame físico (com achado de sopro sistólico, pulsos periféricos amplos e precórdio hiperativo), ecocardiograma e, excepcionalmente, aortografia retrógada. Quando o canal patente foi diagnosticado, e considerado importate, o tratamento clínico foi instituído com restrição de líqüidos, diuréticos e, mais raramente, o uso de digitálicos. Se os sinais começam a regredir e a situação respiratória e clínica melhora após um período de 24 horas, o tratamento clínico é mantido. Caso contrário, o diagnóstico de canal é confirmado e a interrupção é indicada ${ }^{31}$.

A indicação da forma de interrupção, se cirúrgica atendidos por vários grupos de cardiologistas pediatras, atentidos por vários grupos de cardiologistas pediatras, diferentes protocolos foram seguidos. Os principais protocolos foram: 1) indicação primária de interrupção cirúrgica do canal quando presente e importante no prematuro com SAR; 2) tratamento clínico por 24 horas e uma série de 3 doses de indometacina por via oral ou venosa $\left(0,2 \mathrm{mg} \cdot \mathrm{kg}^{-1}\right.$ de peso corporal) e indicaçāo de cirurgia, caso o medicamento não seja eficaz; 3 ) tratamento clínico por 48 horas e 1 ou 2 séries de indometacina por via oral ou venosa (na mesma dose). Em um caso, foi usada uma terceira série, por vią venosa. Nesse protocolo, quase sempre a indicação cirúrgica foi feita em última instância e, geralmente, com o paciente em mau estado geral. Os dois protocolos (2 e 3) foram aplicados somente quando não havia contra-indicação para a indometacina. Todos os pacientes do Grupo 2, que tiveram problemas com o uso de indometacina ou quando após as doses indicadas o canal permaneceu patente ou voltou a abrir, passaram para o grupo em que a ligadura foi realizada (Grupo 1).

\section{TÉCNICA CIRÚRGICA}

Todas as medidas, tanto clínicas como cirúrgicas, nesses prematuros, devem ser extremamente precisas. Assim, a ventilação, a administaçăo de fluidos, a manutençāo da temperatura e todo o ato cirúrgico devem ser minuciosos, para garantir o sucesso. No início da nossa experiência, todos os pacientes eram operados no centro cirúrgico. Desde 1983, passamos a operar na própria unidade de terapia intensiva, tendo sido operados, nessas circunstâncias, 31 pts. (64.5\%).

O paciente está sempre com entubação endotraqueal e com assistência ventilatória. A posiçāo do tubo endotraqueal deve ser comprovada por radiografia de tórax, antes da operação. O prematuro deve estar em leito aquecido, ou sobre colchão de água $\left(37,5^{\circ} \mathrm{C}\right)$, quando no centro cirúrgico. Antes de ser anestesiado, é colocado em decúbito lateral direito, com o braço esquerdo 
MEIER, M. A.; JAZBIK, W.; COUTINHO, J. H.; JAZBIK, J. C.; OLIVEIRA, J. A.; SILVA, J. C.; BARBOSA, R. C.; PAUPÉRIO, H.; SERRA Jr., A. - Manuseio do canal arterial patente no prematuro com síndrome de angústia respiratória: ligadura ou indometacina? Rev. Bras. Cir. Cardiovasc., 4(1): 9-20, 1989.

sobre a cabeça, com uma compressa pequena sob o tórax e fixado nessa poșção com fitas de papel adesivo. Somente depois de todos os preparos de antissepsia e colocados os campos cirúrgicos, é administrado o agente anestésico. A anestesia consiste em ventilação manual sem reinalação com o Baraka e administraçāo de halotano com oxigênio. Não se utiliza nenhum relaxante muscular.

Uma incisāo é feita paralelamente às bordas posterior e inferior da omoplata. Com o braço na posição descrita acima, a omoplata forma, com a coluna vertebral, um ângulo de aproximadamente $45^{\circ}$ (Fig. 1). A toracotomia deve ser pequena, mesmo para o tamanho do paciente, e com preservação completa dos músculos serratus anterior e latissimus dorsi ${ }^{4}$. Iniciando-se no triângulo auscultatório, esses dois músculos sâo parcialmente isolados e afastados, o primeiro anteriormente e o segundo posteriormente, sem seccioná-los. Em seguida, os intercostais são divulsionados com duas pinças de Halsted. Um afastador autostático de Weitlaner pequeno e com dentes rombos (Fig. 2) é colocado entre

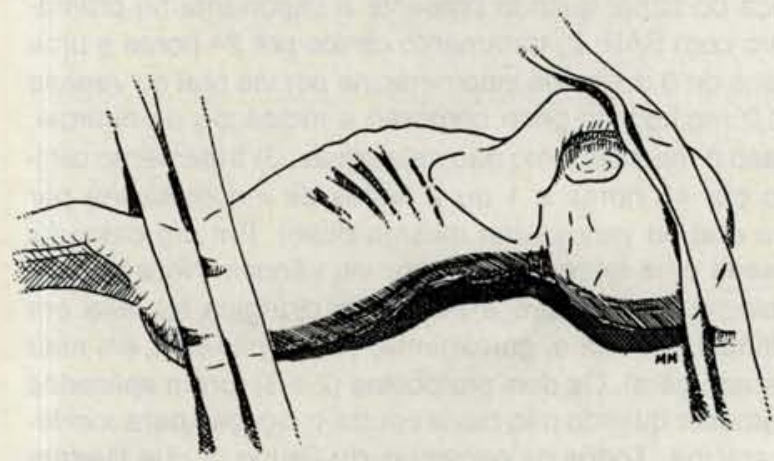

Fig. 1 - Incisão paralela à borda posterior e inferior da omoplata.

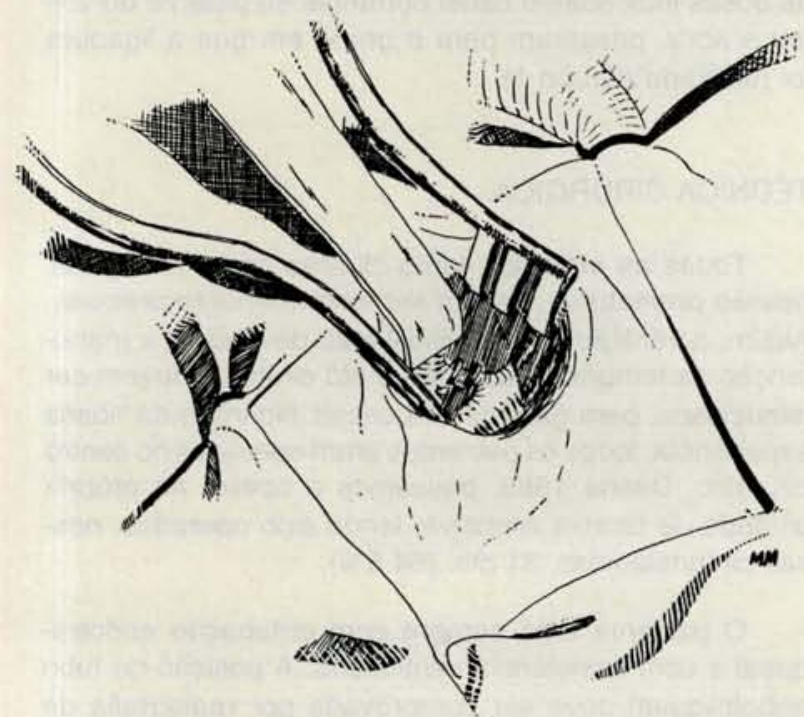

Fig. 2 - Abertura do tórax por afastador autostático e afastamento da pleura e do pulmăo $\mathrm{E}$.

\footnotetext{
- Ethicon L-200.
}

a 3: e 4: costelas e suavemente aberto, procurando afastar as costelas sem abrir a pleura. Uma vez conseguido um afastamento de uns 25 a $35 \mathrm{~mm}$, com um afastador maleável pequeno (10 $\mathrm{mm}$ de largura), a pleura e o pulmão são progressivamente deslocados anteriormente. A pleura parietal se afasta facilmente da parede, sem romper-se. A aorta descendente é localizada e o afastador é dirigido para a regiāo do canal e, com um pouco de tração suave, o canal é alcançado. Os nervos vago e recorrente esquerdos são identificados e afastados anteriormente. $\mathrm{O}$ canal deve ser identificado com segurança, pois, no prematuro, todas as estruturas são muito pequenas, próximas, de diâmetro praticamente iguais e em posiçōes variáveis, o que torna possível a ligadura inadvertida e acidental da artéria pulmonar esquerda, ou mesmo a parte distal do arco aórtico, com graves conseqüências . O canal é, então, levantado com uma pequena pinça de dissecção vascular e interrompido com a aplicação de 2 ou 3 clips metálicos médios*. O primeiro junto da aorta descendente e o segundo próximo à artéria pulmonar (Fig. 3). A dissecção completa e o reparo do canal nāo sāo indicados $24,33,54$; essas manobras são perigosas, devido à fragilidade e à diminuta espessura das paredes do vaso. Uma vez o canal interrompido, a hemostasia é verificada, os afastadores maleável e autostático são retirados.

Em uma primeira série, rotineiramente drenamos o tórax. Numa segunda série, somente os casos em que havia perfuração da pleura. Mais recentemente, somente drenamos o tórax quando há pneumotórax prévio. As costelas são aproximadas com um único ponto de categute cromado 3-0 nos pequenos e 2 pontos 2-0 nos maiores. Quando as costelas são aproximadas, o anestesista mantém uma pressão contínua para expandir os pulmōes e evitar o pneumotórax. Os músculos são aproximados com 3 ou 4 pontos separados de categute simples 4-0 e a pele, fechada com uma sutura intradérmica de

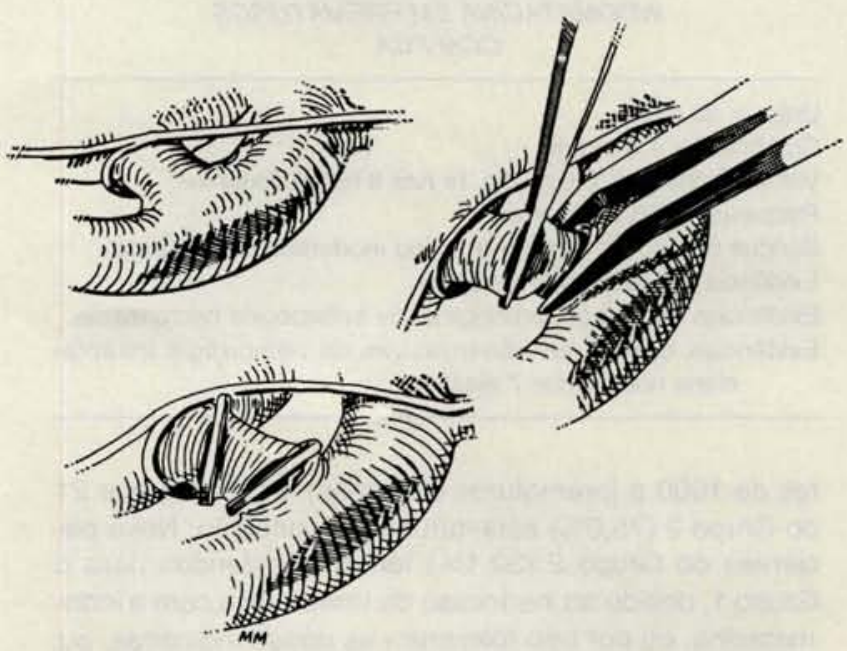

Fig. 3 - Dissecção e colocação do clips no canal. 
MEIER, M. A.; JAZBIK, W.; COUTINHO, J. H.; JAZBIK, J. C.; OLIVEIRA, J. A.; SILVA, J. C.; BARBOSA, R. C.; PAUPÉRIO, H.; SERRA Jr., A. - Manuseio do canal arterial patente no prematuro com síndrome de angústia respiratória: ligadura ou indometacina? Rev. Bras. Cir. Cardiovasc., 4(1): 9-20, 1989.

Mononylon 5-0. Após o curativo, o prematuro é liberado das fitas adesivas, mudado de posição e volta para o ventilador mecânico no próprio leito. Caso a cirurgia tenha sido realizada na sala de operaçōes, o transporte é feito com incubadora portátil.

O prematuro nunca é paralizado com relaxantes musculares, durante a operação. Na grande maioria das vezes, logo após a mudança de posição, já estão com a movimentação normal e pode ser iniciado o processo de desmame do ventilador.

\section{RESULTADOS}

Trinta e nove pacientes do Grupo 1 sobreviveram à operação. A mortalidade, neste grupo, foi de $18,75 \%$ (9 pts.) e, no Grupo 2, de 25,0\% (7 pts.). Quatro (44,4\%) pacientes do Grupo 2 transferidos para o Grupo 1 faleceram. Nenhum óbito foi atribuído ao ato cirúrgico. As causas de óbito, no Grupo 1, foram: sepsis (6 pts.) e hemorragia intracraniana (3pts.). Desses 9 pacientes, 8 pesavam menos de $1000 \mathrm{~g}$. A mortalidade do Grupo 2 foi atribuída a um ou vários fatores; como: sepsis (2 pts.), enterocolite necrotizante (4 pts.), coagulopatia (2 pts.), displasia broncopulmonar (1 pt.), hemorragia intracraniana (2 pts.) e pneumotórax (1 pt.). Todos pesavam menos de $1000 \mathrm{~g}$. A mortalidade dos prematuros extremos (< de $1000 \mathrm{~g}$ ) no Grupo 1 . foi de $24,2 \%$, enquanto que, acima deste peso, houve 1 óbito $(6,6 \%)$. No Grupo 2, a mortalidade dos tratados com peso abaixo dos 1000 g foi de $47,6 \%$, não tendo havido óbitos nos pacientes acima deste peso. Essa diferença é significativa estatisticamente para os dois grupos $(p<0,02)$.

Os óbitos ocorreram entre o 4: e 81: dias de pós-operatório, ou após a administração de indometacina.

As complicações pré-operatórias foram um fator de risco importante. Assim é que, nos 6 pacientes que apresentaram pneumotórax no período pré-operatório, 4 não sobreviveram $(66,6 \%)$. No Grupo 1 , foi, também, um fator de risco o fato de ter o prematuro recebido indometacina nas 72 horas que precederam a cirurgia. Dos 9 pacientes transferidos do Grupo 2 para o Grupo 1 , 4 foram operados nas primeiras 72 horas depois das doses de indometacina. Três pacientes nessas condiçōes faleceram, 1 com coagulopatia e 2 com enterocolite necrotizante. Dois outros pacientes tiveram coagulopatia, muito provavelmente devido à indometacina, e tiveram sangramento excessivo no pós-operatório imediato. Um deles apresentou uma coagulopatia extremamente grave, perdendo 4 vezes o seu volume sangüineo em 24 horas. Esses 2 pacientes sobreviveram.

O seguimento pós-operatório a médio prazo foi sofrível. Dos pacientes do Grupo 1, apenas $20(41,6 \%)$ foram acompanhados por mais de 18 meses, enquanto que, do Grupo 2, foram seguidos 21 (75,0\%). Duas complicações foram mais freqüentes nesses pacientes seguidos: a fibroplasia retrolenticular e distúrbios neurológicos. A primeira complicação ocorreu em 2 pts. do Grupo 1 e 1 pt. do Grupo 2. Distúrbios neurológicos moderados foram diagnosticados em 2 pts. do Grupo 1 e 2 pts. do Grupo 2.

\section{DISCUSSĀO}

Com a introdução da indometacina para o fechamento do canal arterial no prematuro, teve início uma controvérsia ainda não resolvida definitivamente ${ }^{14}$. 15. As vantagens da ligadura cirúrgica são a baixa morbidade e mortalidade e orápido e definitivo fechamento do canal. As desvantagens apregoadas são os riscos inerentes à operação, as complicações infreqüentes, porém possíveis, e os potencialmente deletérios efeitos da toracotomia a longo prazo ${ }^{17,{ }^{37}}$. A grande vantagem do uso da indometacina, para muitos autores ${ }^{60}, 62$, seria interromper o canal arterial, evitando a cirurgia. As desvantagens são a impossibilidade de uso em presença de reduzida função renal, sepsis, coagulopatia, hemorragia intracraniana; provável redução do efeito quando é usada em prematuros extremos; fechamento temporário com reabertura posterior em $25-30 \%$ dos $\operatorname{casos}^{23}$. 39 , além do fato de produzir insuficiência renal, enterocolite necrotizante, perfuração intestinal, coagulopatias e efeitos indesejáveis a longo prazo'.

O manuseio farmacológico do canal arterial baseado no trabalho experimental realizado em várias espécies de animais sugere que, durante a vida fetal, o canal arterial é mantido patente pelas prostaglandinas (PG). A $P E_{2}$ que é produzida na própria parede do canal arterial é, provavelmente, a principal responsável por esse mecanismo; contudo, outras substâncias, como a $\mathrm{PGI}_{2}$, também contribuem. A abertura do canal arterial com o uso de PG foi descrita pela primeira vez em $1975^{19}$, enquanto que o fechamento do canal pelo uso de um inibidor foi relatado em $1976^{19}, 20,26,27$.

O fechamento funcional do canal após o nascimento se inicia com o aumento da saturação de oxigênio do sangue sistêmico que ocorre logo após os primeiros movimentos respiratórios. A velocidade do fechamento varia conforme a espécie, mas, no ser humano, o processo pode levar várias horas para se completar ${ }^{28}$. ${ }^{43}$. Entretanto, em condições normais, mesmo com o canal ainda aberto, ofluxo é pequeno e apenas na direção sistêmicopulmonar. A ação constrictora do oxigênio pode ser complementada por uma redução da sensibilidade do músculo liso do canal às PG's. Em presença de defeitos congênitos do coração que produzam cianose, $O$ aumento normal do $\mathrm{PO}_{2}$ arterial não ocorre $\mathrm{e}$, nessas circunstâncias, freqüentemente $o$ canal demora mais a se fechar. O fechamento parcial ou total que ocorre nessas crianças, 24 a 48 horas depois do nascimento, coincide com o agravamento acentuado que apresentam. Isto também sugere que o fechamento do canal não é total- 
MEIER, M. A.; JAZBIK, W.; COUTINHO, J. H.; JAZBIK, J. C.; OLIVEIRA, J. A.; SILVA, J. C.; BARBOSA, R. C.; PAUPÉRIO, H.; SERRA Jr., A. - Manuseio do canal arterial patente no prematuro com síndrome de angústia respiratória: ligadura ou indometacina? Rev. Bras. Cir. Cardiovasc., 4(1): 9-20, 1989.

mente dependente da oxigenação do sangue. Experimentos realizados com tecidos de canal arterial de vários animais prematuros indicam que o canal do prematuro, se comparado com fetos a termo, é menos sensivel à ação contrátil do oxigênio. Por outro lado, é mais sensivel à ação relaxante das prostaglandinas e também ao efeito contrátil da inibição da síntese dessas substâncias pela indometacina ${ }^{10}$.

A indometacina inibe a ciclo-oxigenase, que é a enzima que converte o precursor, ácido araquidônico, em prostaglandina. Assim, a indometacina evita, não somente a formação de $\mathrm{PGE}_{2}$, mas de todas as PG's, inclusive a $\mathrm{PGI}_{2}$ (prostaciclina), como também algumas substâncias distintas como a Tromboxane $\mathrm{A}_{2}$. A ciclo-oxigenase é encontrada em todos os tecidos do ser humano e não é surpreendente constatar que a sua inibição produz efeitos gerais. Entretanto, a sensibilidade aos efeitos da indometacina varia de tecido para tecido. $O$ importante é que os efeitos constrictores da musculatura lisa do canal arterial é maior nos prematuros ${ }^{34}$.

Durante os últimos anos, vários trabalhos têm descrito o uso clínico da indometacina como alterantiva para a cirurgia, no fechamento do canal arterial em prematuros. A indometacina vem sendo administrada por via oral, por via retal e, mais recentemente, por via endovenosa. É aplicada, geralmente, em dose de 0,1-0,2 mg. $\mathrm{kg}^{1}$, repetida 3 vezes com intervalos de 8 horas. Os resultados positivos têm sido variáveis, entre 18 a $85 \%$, quando usada por via oral ou retal, e de $80-90 \%$, quando aplicada por via endovenosa ${ }^{23,55,62}$. Resultados menos animadores também foram publicdos ${ }^{47}$. 50. McARTHY et alii ${ }^{38}$ publicaram que a resposta à indometacina é dependente da idade gestacional e sugeriram que a resposta não ocorre depois de uma idade correspondente ao equivalente a 36 semanas. Por outro lado, parece existir, também, uma resposta reduzida quando a prematuridade é extrema ${ }^{2}, 11,21,29,48$.

$\mathrm{Na}$ impossibilidade de um estudo retrospectivo e randomizado, e possuidores de uma experiência prévia razoável com os 2 tipos de terapêutica, decidimos fazer apenas um estudo comparativo. Os 2 grupos de pacientes que constituem este estudo foram provenientes de vários berçários e enviados para 7 centros de tratamento intensivo de prematuros. Esta é a razão da diversidade de protocolos seguidos na indicação do tratamento do canal associado à SAR. O nosso objetivo limitou-se a comparar os resultados dos pacientes operados com os que receberam indometacina. Provavelmente, teria sido importante a comparação entre os 3 diferentes protocolos descritos acima, porém, isto foi impossível, devido a uma série de fatores, entre eles as transferências de pacientes entre os vários protocolos e as deficiências encontradas em muitos prontuários. Devido a estes fatos, foram excluídos do estudo outros 13 pacientes, 5 cirúrgicos e 8 tratados com a indometacina.
O estudo desses pacientes, retrospectivo e, obviamente, não randomizado, mostrou que a incidência de enterocolite necrotizante e mortalidade é significativamente menor nos submetidos a ligadura cirúrgica, quando comparados aos que receberam indometacina. Provavelmente, somente este fato não será argumento suficiente para o abandono do uso da indometacina com o objetivo de fechar o canal arterial. Alguns autores atribuem, com razão parcial, esta complicação e a mortalidade à prematuridade ${ }^{23}$. Outros autores, entretanto, publicaram resultados superiores do tratamento cirúrgico, como os de MAHONEY et alii ${ }^{36}$, de MERRIT et alii ${ }^{40}$, de NEU et alii ${ }^{49}$, e YEH et alii ${ }^{61}$. Deve ser enfatizado que, na série cirúrgica, foram operados todos os pacientes com canal arterial patente, mesmo os $40 \%$ que apresentavam complicaçōes pré-operatórias, enquanto que, nos pacientes que receberam indometacina, foram excluídos, ou indicados para cirurgia, os portadores dessas mesmas complicaçōes, como insuficiência renal, sepsis, coagulopatia, hemorragia intracraniana e insuficiênci hepática.

A indometacina é uma droga extremamente tóxica e, por isso, deve ser contra-indicada quando o prematuro apresenta qualquer complicação. Além disso, em geral os trabalhos comparativos ou exclusivamente sobre a indometacina levam em conta os casos de reabertura do canal após um fechamento inicial, o que ocorre com certa frequeência $(25-30 \%)^{8}$, 9, 23, 39. MAHONEY et alli ${ }^{36}$ relatam que o tratamento profilático foi efetivo em pacientes abaixo de $1000 \mathrm{~g}$, porém muito menos efetivo nos acima deste peso.

Os resultados da nossa casuística são muito semeIhantes aos obtidos em outros Centros ${ }^{5,37,42}$. Pacientes pesando menos de $1000 \mathrm{~g}$ tiveram mortalidade muito mais elevada, período de assistência ventilatória muito maior e taxas mais elevadas de complicaçōes. Nos pacientes acima de $1000 \mathrm{~g}$, não houve mortalidade e o número de complicaçōes foi menor. A presença de algumas complicações pré-operatórias foi um fator de risco muito grande. Os pacientes que tiveram coagulopatias e pneumotórax pré-operatórios tiveram mortalidade muito alta.

Os aspectos técnicos da operação merecem algumas consideraçōes. Atualmente, damos preferência por realizar a maioria dessas operaçōes na própria unidade de terapia intensiva. Isto diminui, grandemente, os riscos do transporte, tais como a interrupção da monitorização, instabilidade térmica e ventilação inadequada ${ }^{16}, 51,52$. Por ser uma intervenção de curta duração e realizada por pessoal reduzido, é feita, com segurança e tranqüilidade, na prória CTI, sem interferir com os demais pacientes da unidade. A ventilação é realizada pelo anestesista, com o sistema simples, sem necessidade de desiocar nenhum aparelho do centro cirúrgico. $\mathrm{O}$ paciente é mantido no mesmo leito aquecido e permanece com a mesma monitorização. A operação é realizda pelo cirur- 
MEIER, M. A.; JAZBIK, W.; COUTINHO, J. H.; JAZBIK, J. C.; OLIVEIRA, J. A.; SILVA, J. C.; BARBOSA, R. C.; PAUPÉRIO, H.; SERRA Jr., A. - Manuseio do canal arterial patente no prematuro com síndrome de angústia respiratória: ligadura ou indometacina? Rev. Bras. Cir. Cardiovasc., 4(1): 9-20, 1989.

gião, com apenas um auxiliar e sem instrumentador. Uma vez terminada, basta liberar o paciente das fitas que o sujeitam, colocá-lo em posição supina e tem início o pós-operatório.

A incisāo tem uma importância fundamental, a nosso ver. As toracotomias póstero-laterais, com secção de grandes músculos, são dolorosas, demoram a cicatrizar e causam, sempre, graus variáveis de incompetência funcional imediata e também tardia. Quando essa toracotomia é realizada muito cedo na vida, pode, inclusive, causar deformidade torácica. Com a incisāo posterior e a preservação dos grandes músculos serratus anterior e latissimus dorsi, esses problemas são grandemente minimizados ${ }^{4}$.

O canal pode ser abordado, tanto por via extrapleural, como com abertura da pleura. Preferimos o acesso extrapleural, porque evita o traumatismo pulmonar e reduz a necessidade de drenagem.

Advogamos, enfaticamente, o uso de clips metálicos para a ligadura do canal ${ }^{57}$. Além de ser uma técnica muito mais rápida, mais segura, evita a dissecção e laçada do canal. No prematuro, todas essas estruturas são extremamente delicadas e friáveis, o que torna possível um acidente. Esses pacientes, em geral, sāo hemodinamicamente instáveis e reagem com bradicardia ao mínimo manuseio. As coagulopatias são comuns, quer pela prematuridade, quer pelo emprego prévio de indometacina e qualquer dissecção é, quase sempre, acompanhada de sangramento de difícil controle. Com o uso dos clips com o aplicador adequado, a dissecção e isolamento do canal tornam-se desnecessários, o sangramento é mínimo, ou inexistente. Basta levantar a estrutura ligeiramente com uma pinça de dissecção vascular e aplicar 2 ou 3 clips metálicos médios.

No início da nossa experiência, drenávamos todos os pacientes operados. Com a adoção da técnica de abordar o canal por via extrapleural, passamos a drenar apenas aqueles em que a pleura era acidentalmente perfurada. Ultimamente, a drenagem é empregada apenas quando há pneumotórax esquerdo prévio, ou sangramento excessivo que não pode ser controlado. Nenhum paciente deixado sem dreno teve que ser drenado no pós-operatório, quer por pneumotórax ou por hemotórax. Tivemos que reabrir um prematuro com coagulopatia e sangramento importante, mas que havia sido drenado durante a operação: Cremos que a redução dos drenos diminui a chance de infecção, de pneumotórax e de problemas de manuseio do prematuro ${ }^{5,37,42}$.

Na nossa casuística, não temos um grupo de pacientes tratados apenas clinicamente, para efeitos de comparação, porém a média de dias que os pacientes permaneceram entubados foi de 7,2 dias no Grupo 1 e de 9,8 dias no Grupo 2. Se subdividirmos os 2 grupos em prematuros e prematuros extremos, vamos ver que, no primeiro subgrupo (prematuros), a média foi de 3,9 dias e, no segundo, de 11,4 dias. No Grupo 2, as duas médias foram de 6,8 e 14,7, respectivamente. Todas estas médias incluem os dias pré-operatórios e pré-indometacina. A média de dias pré-operatórios foi de 4,5 dias para o Grupo 1. Estes dados não foram obtidos para o Grupo 2. Se compararmos com as médias dos tratados clinicamente relatados na literatura $(11,2-25,0 \text { dias })^{42}$, veremos que há uma redução acentuada nos prematuros tratados, tanto pela cirurgia, como pela indometacina. Ainda na nossa experiência, aqueles com ligadura tiveram um tempo de entubação menor do que os tratados com indometacina.

Não resta dúvida de que o tratamento cirúrgico, nesses pacientes, é significativamente superior aos obtidos apenas com o tratamento clínico ${ }^{58,60}$. Mesmo nos prematuros em que a insuficiência cardíaca é controlada pela redução da administração de líqüidos e pelo emprego de diuréticos, o tempo de assistência ventilatória é mais prolongado do que nos pacientes nas mesmas condições, nos quais é ligado o canal ${ }^{22,42}$. A toxicidade do oxigênio, entubação endotraqueal com pressão positiva e insuficiência cardiaca secundária ao PCA, todos têm sido implicados na etiopatogenia da displasia broncopulmonar $^{6}$. Na nossa casuística, a ligadura do canal precocemente reduz, significativamente, o tempo de assistência ventilatória. Portanto, reduz a incidência de doenças pulmonares. Além disso, a ligadura do PCA, reduzindo o tempo de assistência ventilatória e, conseqüentemente, o tempo de internação, reduz os custos hospitalares, mesmo levando-se em conta os gastos da operação ${ }^{12,13}$

A cirurgia não acrescenta morbidade e nem mortalidade aos prematuros ${ }^{41}$. As complicações dos casos com ligadura são, de um modo geral, as mesmas complicaçōes da prematuridade e não obrigatoriamente decorrentes da operação. A hemorragia intracraniana, os distúrbios de coagulação e a sepsis são complicações características da prematuridade ${ }^{5,35}$.

O acompanhamento tardio foi bastante prejudicado, devido ao fato de um grande número desses pacientes ser originário de outros Estados e, também, devido ao baixo nível sócio-econômico de parte dessa população. Nos pacientes acompanhados, os resultados foram bastante animadores. A grande maioria se encontra bem. Seis pacientes que já atingiram a idade escolar apresentam desempenho normal. Os distúrbios neurológicos constatados no pequeno número mencionado são leves e moderados. As lesões oculares mencionadas também são moderadas e passíveis de correção. No início desta série, éramos um pouco pessimistas quanto aos resultados a longo prazo do tratamento desses prematuros, Mas, com o passar dos anos, podemos constatar que a grande maioria desses prematuros se desenvolve bem, tornando-se crianças normais. 
MEIER, M. A.; JAZBIK, W.; COUTINHO, J. H.; JAZBIK, J. C.; OlIVEIRA, J. A.; SILVA, J. C.; BARBOSA, R. C.; PAUPÉRIO, H.; SERRA Jr., A. - Manuseio do canal arterial patente no prematuro com síndrome de angústia respiratória: ligadura ou indometacina? Rev. Bras. Cir. Cardiovasc., 4(1): 9-20, 1989.

\section{CONCLUSÕES}

A mortalidade e a mobidade, no grupo operado, foram definitivamente menores do que no grupo que recebeu indometacina.

A presença de complicaçōes pré-operatórias aumenta a morbidade e a mortalidade.

Os pacientes que receberam indometacina e foram operados posteriormente tiveram morbidade e mortalidade mais elevadas.

A mortalidade é, significativamente, maior nos prematuros extremos, tanto para operação, como para a administração de indometacina.

A cirurgia reduz o tempo de assistência ventilatória e de internação, reduzindo, conseqüentemente, os custos.
A indometacina fecha o canal em um número grande de pacientes, mas apresenta um número' significativamente maior de complicaçōes.

A toracotomia com preservação de músculos e por via extrapleural é viável e recomendável. O acesso por via extrapleural foi acompanhado de menor número de complicaçōes.

A drenagem somente é necessária quando há pneumotórax prévio, ou quando há sangramento excessivo. Se usada em todos os pacientes, aumenta o número de complicações.

Baseados nesta casuística, somos levados a recomendar um protocolo de indicação cirúrgica para todos os canais arteriais patentes com sinais de insuficiência cardíaca congestiva e SAR, em prematuros e prematuros extremos.

RBCCV 44205-73

MEIER, M. A.; JAZBIK, W.; COUTINHO, J. H.; JAZBIK, J. C.; OLIVEIRA, J. A.; SILVA, J. C.; BARBOSA, R. C.; PAUPÉRIO, H.; SERRA Jr, A. - Management of patent ductus arteriosus in the premature infant: ligation or indomethacin? Rev. Bras. Cir. Cardiovasc., 4(1): 9-20, 1989.

ABSTRACT: Patent ductus arteriosus (PDA) in low-birth-weight premature infants has been associated with congestive heart failure, bronchopulmonary dysplasia, necrotizing enterocolitis, intracranial hemorrhage, and death. Surgical ligation of the PDA in these infants has improved lung compliance, decreased the duration of assisted ventilation, and increased nutritional utilization. After the introduction of indomethacin for the pharmacological interruption of the PDA in the last decade, several studies have been reported which attempted to establish advantages of the pharmacological treatment over the surgical ligature. The purpose of our study is to compare the results obtained in 48 consecutive patients (Group 1) who underwent surgical ligature of the PDA in the last 8 years, with 28 patients (Group 2), who underwent indomethacin therapy. The mean gestational age of Group 1 was $29.13 \pm 2.33$ weeks (range, 24-34 weeks), and $28.39 \pm 2.30$ weeks (range, 25-32 weeks) in Group 2. The mean birth weight was $954.17 \pm 220.86 \mathrm{~g}$ (range, 540-1750 g) in Group 1, and $923.21 \pm 191.74 \mathrm{~g}$ (range $550-1400 \mathrm{~g}$ ) in Group 2. Thirty three $(60.75 \%)$ pts. from Group 1 weighed $1,000 \mathrm{~g}$ or less, and $21 \mathrm{pts}$. from Group $2(75.0 \%)$ weighed less than $1,000 \mathrm{~g}$. Nine pts. (32.1\%) from Group 2 received indomethacin previously and were transferred to Group 1, due to failure to close the PDA or due to drug intolerance. Ligation of the PDA was performed in the neonatology intensive care unit, in 31 pts. $(64.5 \%)$. Usually a small postero-lateral thoracotomy, with preservation of the latissimus dorsi and anterior serratus muscles, with thorax access through the 3rd or 4 th intercostal spaces. The ductus is isolated by an extrapleural path, and ligated with two or three a medium hemoclips. In our early experience pleural drainage was employed routinely in all patients. Later, it was used only when the pleura was inadvertently opened, and more recently pleural drainage was used only in patients who had preexisting pneumothorax or excessive bleeding. The overall mortality in Group 1 was $18.75 \%$ (9 pts.) and in Group 2 was $25.0 \%$ (7 pts.). Four patients from Group 2 who were transferred to Group 1, died. Causes of death in Group 1, included sepsis, intraventricular hemorrhage, necrotizing enterocolitis and focal perforations, and pneumothorax. Patients who weighed more than $1,000 \mathrm{~g}$ had a better survival and a shorter period of assisted ventilation than patients weighing lesis than $1,000 \mathrm{~g}$. Despite the problems of comparison between the two groups, and limited conclusions that can be drawn from a retrospective and non randomized study. we believe that surgical ligature for PDA was associated with significant less morbidity and mortality, than patients treated with indiomethacin.

DESCRIPTORS: patent ductus arteriosus, surgery. 
MEIER, M. A.; JAZBIK, W.; COUTINHO, J. H.; JAZBIK, J. C.; OLIVEIRA, J. A.; SILVA, J. C.; BARBOSA, R. C.; PAUPÉRIO, H.; SERRA Jr., A. - Manuseio do canal arterial patente no prematuro com síndrome de angústia respiratória: ligadura ou indometacina? Rev. Bras. Cir. Cardiovasc., 4(1): 9-20, 1989.

\section{REFERÊNCIAS BIBLIOGRÁFICAS}

1 ALPERT, B. S.; LEWINS, M. J.; ROWLAND, D. W.; GRANT. M. J.; OLLEY, P. M.; SOLDIN, S. J.; SWYER, P. R.; COCEANI, F.; ROWE, R. D. - Plasma indometacin levels in preterm newborn infants with symptomatic patent ductus arteriosus: clinical and echocardiography assessment of response. J. Pediatr., 95: 578-582, 1979.

2 BAYLEN, B. G.; OKATA, H.; IKEGAMI, M.; JACOBS, H. C.; HOBE, A. H.; EMMANOUILIDES, G. C. - Left ventricular performance and regional blood flow before and after ductus arteriosus occlusion in premature lambs treated with surfactant. Circulation, 67: 837-842, 1983.

3 BELL, E. F.; WARBURTON, D.; STONESTREET, B. S.; $\mathrm{OH}, \mathrm{W} .-$ Effect of fluid administration on the development of symptomatic patent ductus arteriosus and congestive hart failure in premature infants. $N$. Engl. J. Med., 302: 598-601, 1980.

4 BETHENCOURT, D. M. \& HOLMES, E. C. - Musclesparing posterolateral thoracotomy. Ann. Thorac. Surg., 45: 337-339, 1988.

5 BRANDT III, B.; MARVIN, W. J.; EHRENHAT, J. L.; HEINTZ, S.; DOTY, D. B. - Ligation of patent ductus arteriosus in premature infants. Ann. Thorac. Surg., 32: $167-172,1981$.

6 BROWN, E. R. - Increased risk of bronchopulmonary displasia in infants with patent ductus arteriosus. J.Pediatr., 95: 865-866, 1979.

7 CLYMAN, R. I. - The role of the patent ductus arteriosus in respiratory distress syndrome. Semin. Perinatol., 8: 293-297, 1984.

8 CLYMAN, R. I. ; CAMPBELL, D.; HEYMANN, M. A.; MAURAY, F. - Persistent responsiveness of the neonatal ductus arteriosus in immature lambs: a possible for reopening of patent ductus arteriosus after indomethacin-induced closure. Circulation, 71: 141-145, 1985.

9 CLYMAN, R. I. \& HEYMAN, M. A. - Pharmacology of the ductus arteriosus. Pediatr. Clin. N. Am., 28: $77-82,1981$

10 COCEANI, F. \& OLLEY, P. M. - Role of prostaglandins, prostacyclin, and thromboxanes in the control of prenatal patency and postnatal closure of the ductus arteriosus. Semin. Perinatol., 4: 109-113, 1980.

11 COOKE, R. W. I. \& PICKERING, D. - Poor response to oral indomethacin therapy for persistent ductus arteriosus in very low birth weight infants. Br. Heart J., 41: $301-303,1979$.

12 COTTON, R. B.; STAHLMAN, M. T.; BENDER, H. W.; GRAHAM T, P.; CATTERTON, W. Z.; ROVAR, I. Randomized trial of early closure of symptomatic patent ductus arteriosus in small preterm infants. J. Pediatr/. 93: $647-651,1978$.
13 COTTON, R. B.; STHALMAN, M. T.; KOVAR, I.; CATTERTON, W. Z. - Medical management of small infants with symptomatic patent ductus arteriosus. $J . P e$ diatr., 92: 467-474, 1978.

14 EDMUNDS Jr., L. H. - Operation or indomethacin for the premature ductus. Ann. Thorac. Surg., 26: 586-569, 1978.

15 EDMUNDS Jr., L. H.; GREGORY, G. A.; HEYMANN, M. A.; KITTERMAN, J. A.; RUDOLPH, A. M.; TOLLEY, W. H. - Surgical closure of the ductus arteriosus in premature infants. Circulation, 48: 856-862, 1973.

16 EGGERT, L. D.; JUNG, A. L.; McGOUGH, E. C.; RUTTENBERG, H. D. - Surgical treatment of patent ductus arteriosus in preterm infants: four-year experience with ligation in the newborn intensive care unit. Pediatr. Cardiol., 2: 15-19, 1982.

17 ELLISON, R. C.; PECKHAM, G. J.; LANG, P.; TALNER, N. S.; LERER, T. J.; LIN, L.; DOOLEY, K. J.; NADAS, A. S. - Evaluation of the preterm infant for patent ductus arteriosus. Pediatrics, 71: 364-370, 1983.

18 FLOWER, R. W. \& BLAKE, D. A. - Retrolental fibroplasia: evidence for a role of the prostaglandin cascade in the pathogenesis of oxygen-induced retinopathy in the newborn beagle. Pediatr. Res., 15: 660-668, 1981.

19 FRIEDMAN, W. F.; HIRSCHKLAU, M. J.; PRINTZ, M. P.; PITLICK, P. T.; KIRKPATRICK, S. E. - Pharmacologic closure of patent ductus arteriosus in the premature infant. N. Engl. J. Med., 295: 526, 1976.

20 FRIEDMAN, W. F.; HIRSCHKLAU, M. J.; PRINTZ, M. P.; PITLICK, P. T.; KIRKKPATRICK, S. E. - Pharmacologic closure of patent ductus arteriosus in hyaline membrane disease. J. Pediatr.. 93: 682-689, 1978.

21 FRIEDMAN, W. F.; KURLINSKI, J.; JACOB, J.; DISESSA, T. G.; CLUCK, L.; MERRIT, T. A.; FELDMAN, B. H. - The inhibition of prostaglandin and prostacyclin synthesis in the clinical management of patent ductus arteriosus. Semin. Perinatol., 4: 125-133, 1980.

22 GAY, J. H.; DAILY, W. J. R.; MEYER, B. H. P.; TRUMP, D. S.; CLOUD, D. T.; MOLTHAN, M. E. - Ligation of the patent ductus arteriosus in premature infants: report of 45 cases. J. Pediatr. Surg., 8: 677-683, 1973.

23 GERSONY, W. M.; PECKHAM, G. J.; ELLISON, R. C.; MIETTINEN, O. S.; NADAS, A. S. - Effects of indomethacin in premature infants with patent ductus arteriosus: results of a national collaborative study. J.Pediatr., 102: 895-906, 1983.

24 GUNNING, A. J. - A simple, safe, surgical technique for closing the persistent ductus arteriosus in the preterm neonate. Ann. A. Coll. Surg. Eng., 65:214-216, 1983.

HARINCK, E.; VAN ERTBRUGGEN, L.; SENDERS, R. C. H.; MOULAERT, A. J. - Problems with indomethacin for ductus closure. Lancet., 2: 245, 1977. 
MEIER, M. A.; JAZBIK, W.; COUTINHO, J. H.; JAZBIK, J. C.; OLIVEIRA, J. A.; SILVA, J. C.; BARBOSA, R. C.; PAUPÉRIO, H.; SERRA Jr., A. - Manuseio do canal arterial patente no prematuro com síndrome de angústia respiratória: ligadura ou indometacina? Rev. Bras. Cir. Cardiovasc., 4(1): 9-20, 1989. patent ductus arteriosus in premature infants. Paediatrician, 7: 3-17, 1978.

27 HEYMANN, M. A.; RUDOLPH, A. M.; SILVERMAN, N. H. - Closure of the ductus arteriosus in premature infants by inhibition of prostaglandin synthesis. N. Engl. J. Med., 295: 530-536, 1976.

HOAFFMAN, J. I. E.; HEYMANN, M. A.; CLYMAN, R. I.; RUDOLPH, A. M. - Patent ductus arteriosus in premature infants. Rev. Latina Cardiol. Infant., 4: 293-306, 1986.

29 IVEY, H.H.; KATTIWINKEL, J.; PARK, T. S.; KROVETZ, L. J. - Failure of indomethacin to close persistent ductus arteriosus in infants weighing under 1000 grams. Br. Heart. J., 41: 305-307, 1979.

JACOB, J.; GLUCK, L.; DI SESSA, T.; EDWARDS, D.; KULOVICH, M.; KURLINSKI, J.; MERRIT, T. A.; FRIEDMAN, W. F. - The contribution of PDA in the neonate with severe RDS. J. Pediatr., 96: 79-85, 1980.

31 JOHNSON, G. L.; BREART, G. L.; GEWITZ, M. H.; BRENNER, J. I.; LANG, P.; DOOLEY, K. J.; ELLISON, R. C. - Echocardiographic characteristics of premature infants with patent ductus arteriosus. Pediatrics., 72 : $846-849,1983$.

32 KITTERMAN, J. A.; EDMUNDS Jr., L. H.; GREGORY, G. A.: HEYMANN, M. A.; TOOLEY, W. H.; RUDOLPH, A. M. - Patent ductus arteriosus in premature infants: incidence, relation to pulmonary disease and management. J. Engl. J. Med., 287: 473-477, 1972.

33 KRON, I. L.; MANTZER Jr., R. M.; RHEUBAN, K. S.; NOLAN, S. P. - A simple rapid technique for operative closure of patent ductus arteriosus in the premature infant. Ann. Thorac. Surg., 37: 422-427, 1984.

34 LEWIS, A. B.; HEYMANN, M. A.; RUDOLPH, A. M. Gestational changes in pulmonary vascular response in fetal lamb in utero. Circ. Res., 39: 536-542, 1976.

35 MAHER, P.; LANE, B.; BALLARD, R.; PIECUCH, R.; CLYMAN, R. I. - Does indomethacin cause extensions of intracranial hemorrhages: a preliminary study. $P e$ diatrics, 75: 497-504, 1985.

36 MAHONEY, L.; CARNERO, V.; BRETT, C.; HEYMANN, M. A.; CLYMAN, R. A. - Prophylactic indomethacin therapy for patent ductus arteriosus in very-low-birthweight infants. N. Engl. J. Med., 306: 506-511, 1982.

37 MAVROUDIS, C.; COOK, L. N.; FLEISCHAKER, J. W. NAGARAJ, H. S.; SHOTT, R. J.; HOWE, W. R.; GRAY Jr., L. A. - Management of patent ductus arteriosus in the premature infant: indomethacin versus ligation. Ann. Thorac. Surg., 36: 561-566, 1983.

38 McCARTHY J. S.; ZIES, L. G.; GELBAND, H. - Age-dependent closure of the patent ductus arteriosus by indomethacin. Pediatrics, 62: 706-712, 1978.
39 MELLANDER, M.; LEHEUP, B.; LINDSTROM, D. R.; PALME, C.; GRAHAM Jr., T. P.; STAHLMAN, M. T.; COTTON, R. B. - Recurrence of symptomatic patent ductus arteriosus in extremely premature infants, treated with indomethacin. J. Pediatr., 105: 138-146, 1984.

MERRITT, T. A.; HARRIS, J. P.; ROGHMANN, K.; WOOD, B.; CAMPANELLA, V.; ALEXSON, C.; MANNING, J.; SHAPIRO, D. L. - Early closure of the patent ductus arteriosus in very low-birth-weight infants: a controled trial. J. Pediatr., 99: 281-288, 1981.

41 MERRITT, T. A.; WHITE, C. L.; JACOB, J.; KURLINSKI, J.; MARTIN, J.; DI SESSA, T. G.; EDWARDS, D.; FRIEDMAN, W. F.; GLUCK, L. - Patent ductus arteriosus treated with ligation or indomethacin: a follow-up study. J. Pediatr., 95: 588-594, 1979.

42 MIKHAIL, M.; LEE, W.; TOEWS, W.; SYNHORST, D. P.; HAWES, C. R.; HERNANDEZ, J.; LOCKHART, C.; WHITFIELD, J.; PAPPAS, G. - Surgical and medical experience with 734 premature infants with patent ductus arteriosus. J. Thorac. Cardiovasc. Surg., 83: 349-357, 1982.

43 MOMMA, K.; KONISHI, T.; HAGIWARA, H. - Characteristic morphology of the constricted fetal ductus arteriosus following maternal administration of indomethacin. Pediatr. Res., 19: 493-499, 1985.

44 NADAS, A. S. - Patent ductus revisited. N. Engl. J. Med., 295: 563, 1976.

45 NAGARAJ, H. S.; SANDHU, A. S.; COOK, L. - Gastrointestinal perforation following indomethacin therapy in very low birth weight infants. J. Pediatr. Surg., 16: 1003-1009, 1981.

46 NAULTI, C. M; HORN, S.; CONRY, J.; AVERY, G. B. Improved lung compliance after ligation of patent ductus arteriosus in hyaline membrane disease. J.Pediatr., 93: 682-689, 1978.

47 NEAL, W. A.; BESSINGER Jr., F. B.; HUNT, C. E.; LUCAS Jr., R. V. - Patent ductus arteriosus complicating respiratory distress syndrome. J. Pediatr., 86: 127-136, 1975.

48 NEAL, W. A.; LYLE, J. M.; MULLET, M. D. - Failure of indomethacin therapy to induce closure of patent ductus arteriosus in premature infants with respiratory distress syndrome. J. Pediatr., 91: 621-623, 1977.

49 NEU, J.; ARIAGNO, R. L.; JOHNSON, J. B. - A doubleblind study of the effects of oral indomethacin in preterm infants with patent ductus arteriosus who failed medical management. Pediatr. Pharmacol., 1: 245-250, 1981.

50 OLLEY, P. M.; COCEANI, F.; FREEDOM, R. M.; IZUKAWA, T.; SWYER, P. R.; ROWE, R. D. - Pharmacological manipulations of the ductus arteriosus. In: GODMAN, M. J. (ed.) Paediatric cardiology. Edinburgh, Churchill Livingstone, 1981 . Vol. 4, p. 259-266. 
MEIER, M. A.; JAZBIK, W.; COUTINHO, J. H.; JAZBIK, J. C.; OLIVEIRA, J. A.; SILVA, J. C.; BARBOSA, R. C.; PAUPÉRIO, H.; SERRA Jr., A. - Manuseio do canal arterial patente no prematuro com síndrome de angústia respiratória: ligadura ou indometacina? Rev. Bras. Cir. Cardiovasc., 4(1): 9-20, 1989.

51 OXNARD, S. C.; MCGOUCH, E. C.; JUNG, A. L.; RUTTENBERG, H. D. - Ligation of the patent ductus arteriosus in the newborn intensive care unit. Ann. Thorac. Surg., 23: 564-570, 1977.

SALOMON, N. W.; ANDERSON, R. M.; COPELAN, J. G. - A rational approach to ligation of patent ductus arteriosus in the neonate. Chest, 75: 671-678, 1979.

53 SIASSI, B.; BLANCO, C.; CABAL, L. A.; CORAN, A. G. - Incidence and clinical features of patent ductus arteriosus in low-birth-weight infants: a prospective analysis of 150 consecutively born infants. Pediatrics, 57: 347-361, 1976.

54 STRANGE, M.; MYERS, G.; KIRKLIN, J. K.; PACIFICO, A. D.: CASSADY, G. - Lack of effect of patent ductus arteriosus ligations on intraventricular hemorrhage in preterm infants. Clin. Res., 31: 913, 1983.

55 THALJI, A. A.; CARR, I.; YEH, T. F.; RAVAL, D.; LUKEN, J. A.; PILDES, R. S. - Pharmacokinetics of intravenously administered indomethacin in premature infants. J. Pediatr., 87: 995-1001, 1980.

56 THIBEAULT, D. W.; EMMANOUILIDES, G. C.; NELSON, R. J.; LACHMAN, R. S.; ROSENGARDT, R. M.; OH, W. - Patent ductus arteriosus complicating the respiratory distress syndrome in preterm infants. J. Pediatr., 86: 120-129, 1975.

57 TRAUGOTT, R. C.; WILL, R. J.; SCHUCHMANN, G. F.; TREASURE, R. L. - A simplified method of ligation of patent ductus arteriosus in premature infants. Ann. Thorac. Surg., 29: 263-267, 1980.

58 WAGNER, H. R.; ELLISON, R. C.; ZIERLER, S.; LANG, P.; PUROHIT, D. M.; BEHRENDT, D.; WALDHAUSEN, J. A. - Surgical closure of patent ductus arteriosus in 268 preterm infants. J. Thorac. Cardiovasc. Surg., 87: 870-878, 1984.

59 WAY, G. L.; PIERCE, J. R.; WOLFE, R. R.: MCGRATH, R.; WIGGINS, J.; MERENSTEIN, J. B. - ST depression suggesting subendocardial ischemia in neonates with respiratory distress syndrome and patent ductus arteriosus. J. Pediatr., 95: 609-614, 1978.

YANAGI, R. M.; WILSON, A.; NEWFELD, E. A.; AZIZ, K. V.; HUNT, C. E. - Indomethacin treatment for symptomatic patent ductus arteriosus: a double-blind control study. Pediatrics, 67: 647-656, 1981

61 YEH, T. F.; LUKEN, J. A.; THALJI, A.; RAVAL, D.; CARR, I.; PILDES, R. S. -- Intravenous indomethacin therapy in premature infants with persistent ductus arteriosus a double-blind study. J. Pediatr., 98: 137-146, 1981.

62 YEH, T. F.; THALJI, A.; LUKEN, J.; RAVAL, D.; CARR I.; PILDES, R. S. - Intravenous indomethacin therapy in premature infants with PDA: a double blind control study. Pediatr. Res., 13: 354-360, 1979.
63 ZACHMAN, R. D.; STEINMETZ, G. P.; BOTHAM, R. J.; GRAVEN, S. N.; LEDBETTER, M. K. - Indicence and treatment of the patent ductus arteriosus in the ill premature neonate. Am. Heart J., 87: 697-702, 1974.

\section{Discussão}

\section{DR. FÁBIO SALLUM \\ Curitiba, $P R$}

Gostaria, inicialmente, de agradecer à Comissāo Organizadora, a oportunidade de comentar este trabalho, que questiona, de maneira comparativa e retrospectiva, o uso da ligadura cirúrgica, ou da administração da indometacina para o fechamento da persistência do canal arterial em prematuros. Este é um tema polêmico e ainda não inteiramente definido na literatura. Trabalhos publicados em 1979 reviram 52 pacientes com 26 ligaduras e 26 tratamentos com indometacina e não encontraram diferenças estatisticamente significativas de sobrevida, que foi, nos dois grupos, de $81 \%$. Em nossa experiência, no Hospital Infantil Pequeno Príncipe, de Curitiba, em cerca de 2.000 crianças operadas, ligamos o canal arterial em 323 ocasiōes, sendo em torno de 100 casos abaixo do primeiro ano de vida, mas apenas em 2 prematuros; outros casos chegados ao Hospital foram fechados com indometacina, ou aguardou-se a própria evolução natural com fechamento expontâneo, experiência esta superponivel à do $\mathrm{InCor}$, conforme nos relatou o $\mathrm{Dr}$. Miguel Barbero-Marcial. Gostaria de ressaltar o trabalho de Garson e colaboradores, em 1983, sobre um estudo duplo-cego cooperativo em 13 Instituiçōes americanas, com 421 pacientes, onde o canal arterial fechou expontaneamente em prematuros. O Dr. Meier dividiu sua casuística em 2 grupos; para nōs, foi difícil a fiel interpretação dos números apresentados, já que um mesmo grupo apresentava pacientes submetidos a diferentes tratamentos; imaginamos, então, uma alteração metodológica, com a formação de 3 grupos, que chamaríamos Grupo A: pacientes submetidos somente a ligadura, 39; Grupo B: pacientes submetidos a indometacina e ligadura, 9; Grupo C: pacientes submetidos somente a indometacina, 19. Submetemos esses 3 grupos ao teste de significância estatística e vimos que os resultados obtidos pelo Grupo A (somente ligadura) foram a priori significativamente superiores. Seria importante, entretanto, neste trabalho do Dr. Meier, a análise estratificada de cada grupo em relação, por exemplo, ao peso e à idade gestacional, para podermos correlacionar os resultados, não somente ao tipo de tratamento, mas também ao grau de prematuridade do grupo. A medida do átrio esquerdo pelo ecocardiograma, na série, poderia, também, adicionar mais unidade para a comparaçāo entre os grupos, de forma mais homogênea. O Dr. Meier concluiu que, na sua casuística, pacientes submetidos à indometacina apresentaram maior número de complicaçōes e taxa de mortalidade mais elevada; sabemos que os efei- 
MEIER, M. A.; JAZBIK, W.; COUTINHO, J. H.; JAZBIK, J. C.; OLIVEIRA, J. A.; SILVA, J. C.; BARBOSA, R. C.; PAUPÉRIO, H.; SERRA Jr., A. - Manuseio do canal arterial patente no prematuro com síndrome de angústia respiratória: ligadura ou indometacina? Rev. Bras. Cir. Cardiovasc., 4(1): 9-20, 1989.

tos colaterais da indometacina têm relação dose-idade gestacional intimamente relacionados; como $90 \%$ dos pacientes do chamado Grupo 2 apresentavam menos de 32 semanas, que é o grupo mais susceptivel aos efeitos colaterais, à correlação entre a dose utilizada em relaçāo à idade gestacional, o que seria importante no sentido de determinar algumas causas de óbito no Grupo 2, como hemorragias e distúrbios de coagulação; a análise do Grupo 2 demonstra, também, que apenas $10 \%$ dos pacientes apresentavam idade inferior a 7 dias, que é o período onde a indometacina apresenta a maior eficácia; doses maiores de indometacina em pacientes acima dessa faixa etária podem ter contribuido para um maior número de complicaçōes. Gostaria, finalmente, de perguntar do Dr. Milton qualé a incidência de ligadura de canal arterial em prematuros em relação à sua casuística global com ligadura em pacientes maiores. Obrigado.

\section{DR. RICARDO DE CARVALHO LIMA}

Recife, $P E$

Gostariamos de agradecer a organização deste encontro, pela oportunidade que nos proporciona como comentador oficial de tão interessante trabalho. Sem dúvida, o trabalho aqui apresentado pelo Dr. Milton Meier é original na literatura brasileira e aproveitamos a oportunidade para cumprimentá-lo, juntamente com seus colaboradores. As primeiras descriçōes de fechamento cirúrgico de PCA em crianças prematuras foram feitas por Powell e De Cancq, no Med. J. Aust. 2: 56, 1963, e no Am. J. Dis. Child. 106: 402, 1963, respectivamente. Em geral, a persistência do canal arterial em crianças prematuras apresenta-se com 3 quadros clinicos distintos: 1) PCA sem doença pulmonar: essas crianças apresentam peso ao nascimento acima de $1500 \mathrm{~g} \mathrm{e}$, geralmente, não desenvolvem severa insuficiência ventricular esquerda. São facilmente manuseadas com terapia convencional e, na gande maioria, o PCA fecha, expontaneamente, dentro de 2 a 3 meses após o nascimento. 2) PCA em crianças em fase de recuperação de doença pulmonar (SAR): neste grupo, as crianças apresentam peso entre $1000 \mathrm{~g} \mathrm{e} 1500 \mathrm{~g}$ ao nascimento. A síndrome de angústia respiratória torna-se evidente dentro de pouas horas após o nascimento e a melhora do quadro clínico se inicia após 3 a 4 dias. Com a melhora do quadro clínico e diminuição da resistência vascular pulmonar, inicia-se a evidência de shunt E-D através do canal arterial. Este é o grupo mais comum. 3) PCA com doença pulmonar (SAR); este grupo consiste de crianças que têm severa SAR desde o nascimento, sem melhora do quadro clínico, quando da queda da resistência vascular pulmonar e aumento do shunt E-D. A maioria dessas crianças apresenta, ao nascimento, peso de $1200 \mathrm{~g}$.
No entanto, comparaçōes entre o tratamento médico versus cirúrgico, nos grupos acima, são particularmente dificeis. No trabalho aqui apresentado, foram analisados 2 grupos, onde o peso variou de 540 a 1750 g. Gostaríamos de saber do autor se foi observada a presença dos 3 quadros clínicos distintos em cada um de seus grupos e se houve alguma correlação com os protocolos utilizados versus mortalidade. A prematuridade na presença de PCA apresenta, como grave complicação, a enterocolite necrotizante. A endometacina talvez não seja a responsável isolada por essa complicaçāo e, sim, a isquemia intestinal devida ao grande shunt E-D com falência ventricular esquerda. Depende de vários fatores a decisão de fechar o canal por meios farmacológicos, ou através da ligadura cirúrgica. O mais importante é analisar a mortalidade apresentada pelas duas opçōes. Em um estudo multicêntrico, realizado nos Estados Unidos, segundo Nadas e colaboradores (J. Pediat. 98: 137, 1981), não existiu diferença entre a mortalidade dos pacientes tratados cirurgicamente sem nunca ter recebido indometacina com aqueles que foram tratados com indometacina. A seleçāo da terapia depende, também, de outros fatores, tais como: facilidade de cirurgia, condição geral do paciente e presença de contra-indicaçōes para o uso da indometacina. Acredito que a decisāo deva ser tomada individualmente, para cada caso. Na Unidade de Cardiologia Pediátrica do Hospital Barão de Lucena, no Recife, 7 pacientes, com peso variando entre 980 e $2000 \mathrm{~g}$, foram tratados com indometacina. Foram observados 1 óbito (1/7-14,3\%) e 2 insucessos (2/6-33,3\%). Finalmente, gostariamos de paranebizar os autores, pelo excelente trabalho e por nos trazerem um assunto tão especial e controverso.

\section{DR. MEIER \\ (Encerrando)}

Os pacientes que são tratados clinicamente adequadamente, permanecem, no entanto, na unidade de neopatologia; isto baseados em 7 trabalhos recentes, de 1985 para cá, 37 vezes mais dias do que os que são tratados cirurgicamente; portanto, é óbvio que é um paciente que fica mais caro, que requer muito mais trabalho e a mortalidade dos 2 grupos é absolutamente igual. A literatura vem demonstrando a superioridade da ligadura cirúrgica sobre a indometacina; ontem, convrsávamos com o Dr. Pacífico e ele informou que já a abandonou já faz 3 anos; o grupo do Dr. İber, da Universidade da Califórnia, onde surgiu a indometacina, já a abandonou e, há 2 anos, faz a ligadura direita, sem o emprego de inibidores da prostaglandina. Ao Dr. Ricardo Lima agradeço os comentários. Obrigado. 